\title{
MACHINE LEARNING FOR MULTI-MODALITY GENOMIC SIGNAL PROCESSING
}

\author{
Sun-Yuan Kung and Man-Wai Mak
}

One of the main challenges in computational biology is the revelation and interpretation of the rich genomic information underlying cancer biology. Revealing such information can help facilitate classification and prediction of cancers and responses to therapies. Genomic sequencing and gene expression technologies have been widely recognized as vital approaches to modern drug design and disease classification. A collection of microarray experiments can yield a data matrix, whose rows simultaneously reveal the expression level of tens of thousands of genes, a promising advance towards a genome-wide exploration.

Machine learning has been a promising computational approach to genomic studies. A machine learning system comprises two subsystems: (1) feature extraction and (2) adaptive classification or cluster discovery. For adaptive classifiers, both unsupervised and supervised training strategies have been found useful.

In the past, the prevalent approaches to gene classification relied on only a single modality. However, there is recently increasing evidence of substantial performance improvement accomplished by combining information from multiple sources. This calls for a new classification approach based on multi-modality fusion.

\section{Multi-Modality Fusion for Genomic Signal Processing}

There are many ways to generate multiple modalities, one is via sensor diversity and the other via feature diversity. In terms of sensor diversity, we shall consider both the motif and gene expression modalities. Motifs are short sequences of DNA responsible for regulating gene networks and the expression of genes, whereas gene expression is the process of producing proteins from information coded in genes. A genome-wide study via the pure sequencing approach is computationally prohibitive, thus gene expression analysis can play a vital and complementary role in this respect. To further facilitate multi-modality fusion, a diversity of features may be extracted from each sensor by computational means. This is called feature diversity.

There are two basic fusion architectures—cascaded and parallel—suitable for genomic signal processing. For simplicity, we shall use only two modalities to illustrate the possible fusion strategies. (The extension to the fusion of multiple modalities is straightforward.) The fusion architectures can be mathematically represented as follows:

$$
\begin{aligned}
\text { Cascaded }: f(\gamma(X), Y) & \rightarrow \text { Fusion output } \\
\text { Parallel }: g(\phi(X), \psi(Y)) & \rightarrow \text { Fusion output }
\end{aligned}
$$


where $X$ and $Y$ are the features of the two modalities. Typically, $f$ and $g$ are binary functions (i.e., they either output positive or negative class labels), whereas $\gamma, \phi$, and $\psi$ can be either binary functions or score functions.

Fusion can be viewed as a classification problem in which optimal decision boundaries (c.f. fig 1) are defined to separate the positive and negative patterns in the score space. The effect of fusion can be illustrated by the receiver operating characteristics (ROCs) [4] as shown in fig 2. The performance of cluster discovery and the improvement gained by fusion can be evaluated via its goodness for gene prediction. Specifically, a test gene $t$ will be predicted as positive if

$$
\log p\left(s(t) \mid \Lambda^{(p)}\right)>\log p\left(s(t) \mid \Lambda^{(n)}\right)+\alpha,
$$

where $p\left(s(t) \mid \Lambda^{(p)}\right)$ and $p\left(s(t) \mid \Lambda^{(n)}\right)$ denote the distribution of the feature values $s(t)$ 's being generated by the positive and negative models $\Lambda^{(p)}$ and $\Lambda^{(n)}$, respectively. It is common to adopt the following performance measures: Precision $=\frac{\mathrm{TP}}{\mathrm{TP}+\mathrm{FP}}$, Sensitivity $=\frac{\mathrm{TP}}{\mathrm{TP}+\mathrm{FN}}$, and Specificity $=\frac{\mathrm{TN}}{\mathrm{FP}+\mathrm{TN}}$, where TP, TN, FP, and FN are the numbers of true-positives, true-negatives, false-positives, and false-negatives, respectively. ROCs display the trade-off of two of these measurements over their entire range. To produce the entire spectrum of sensitivity-precision-specificity ROCs, we can gradually adjust the running variable $\alpha$ in Eq. 3 to change from small to large values. For example, by setting $\alpha<0$ (respectively $\alpha>0$ ), we can ob- tain a higher sensitivity (respectively precision).

Let us illustrate an application example of cascaded fusion. Suppose that a gene is classified to be positive if and only if it is admitted by both modalities. Such a classification can be implemented by a cascaded architecture with the ANDlogic in fig 1(a). Suppose the first modality, adopting an exploded search space, yields a result with high sensitivity (due to a large number of true positives) at the expense of poor precision (due to a large number of false positives). Now, according to Eq. 1, the second modality (represented by $f$ ) can be used to further screen all the genes which are admitted to be positive by the first modality (represented by $\gamma$ ). Suppose a far greater number of false positives than true positives can be weeded out, then the precision will be substantially improved while the sensitivity remains very much intact. This process is manifested by the boldfaced arrow depicted in fig 2(a).

Similar argument can be applied to parallel fusion. Suppose that a gene is predicted to be positive as long as it is admitted by either of the two modalities. This can be implemented by using the parallel architecture with the OR-Logic in fig 1(b). The potential improvement of such a fusion scheme is manifested by the boldface arrows in fig 2(b).

The general scheme is that with a parallel architecture, nonlinear fusion leads to nonlinear decision boundaries as illustrated in fig 1(c). All 
the previously mentioned scenarios are just special cases of nonlinear fusion. Moreover, nonlinear fusion is often implemented via a machine learning or neural network approach. Therefore, the machine learning approach provides a unified framework for multi-modality fusion.

\section{Sensor Diversity: A Biological Perspective}

The genome is not just a collection of genes working in isolation, but rather it encompasses the global and highly coordinated control of information to carry out a range of cellular functions. Therefore, it is imperative to conduct genome-wide studies so as to facilitate (1) effective identification of correlated genes and (2) better understanding of the mechanisms underlying gene transcription and regulation.

It is known that within the DNA sequences, there are short segments of DNA called motifs that are responsible for regulating the gene networks and the expression of genes. Therefore, the knowledge of motifs provides important information for biologists to verify and explain experimental results. However, the downside of relying only on gene sequencing (or motif discovery) for genomic scale studies lies in its extremely high computational complexity.

Expression of several thousands of genes can be measured simultaneously by DNA microarrays. The upside is that gene expression analysis is computationally less demanding than sequencing. Furthermore, recent advances in machine learning tools for expression profiling have become more mature and cost effective. However, microarray data are very noisy and contain artifacts, making gene prediction very difficult.

There already exist strong evidences supporting the correlation of motifs and expression profiles $[1,8,9]$. This suggests that these two modalities are complementary to each other, making them legitimate candidates for fusion. Park et al. [8] found genes that have similar promoter regions and determined the extent to which these genes have similar expression profiles. It was concluded that, on the average, genes with similar promoter regions have significantly higher correlation, although the correlation can vary widely depending on genes.

A more promising result supporting the prediction of gene expression from DNA sequences was reported by Beer and Tavazoie [1]. In their work, gene expression patterns were predicted by looking only at their regulatory sequences. For thousands of genes, they found the set of DNA sequence elements most likely to be responsible for their expression. It was concluded that (1) to a very high degree the gene expression is determined by local sequences and (2) the inferred regulatory rules can correctly predict expression patterns for $73 \%$ of genes in yeast.

Conlon et al. [3] proposed a cascaded fusion architecture in which microarray expression values are used to help screen out false positive mo- 
tifs found by a motif discovery searching algorithm called Motif Discovery Scan (MDscan). The strength of correlation between gene expressions and motifs (represented by motif matching scores) is captured by linear regression coefficients, which are determined by a selective regression procedure. A larger nonzero regression coefficient suggests that the corresponding gene expression is more correlated with the upstream sequences containing the motif. This approach is able to increase both the sensitivity and specificity of the discovered motifs. Naturally, the selected motifs and the regression equations can be used to predict other genes in the same group.

In contrast, Kasturi and Acharya [5] adopted a parallel fusion architecture to combine promoter sequences of genes/DNA binding motifs and gene expression profiles. In their work, similarity scores based on the frequency of motif occurrences and expression intensities were probabilistically combined during gene cluster identification. To fuse the information embedded in sequence data and expression data, the Kohonen feature map was adopted to simultaneously update the expression profile clusters and sequence clusters.

\section{Feature Diversity: An Algorithmic \\ Perspective}

Note that it is crucial not only how to cluster data but also how to find an appropriate way of looking at data. Thus, extracting correct features is essential for gene prediction. We now introduce a comprehensive set of coherence models to better capture the biological relevant features of genes to improve prediction performance. Two popular coherence models are the additive coherence model and multiplicative coherence model.

1. Additive coherence model: A scaling relation between $\mathrm{mRNA}_{a}$ and $\mathrm{mRNA}_{b}$ is expressed as mRNA $_{b}=\lambda\left(\mathrm{mRNA}_{a}\right)$, where $\lambda$ is a scaling factor. Denote $a=\log \left(\mathrm{mRNA}_{a}\right)$ and $b=\log \left(\mathrm{mRNA}_{b}\right)$, we have $b=\lambda^{\prime}+a$ where $\lambda^{\prime} \equiv \log (\lambda)$.

2. Multiplicative coherence model: An exponential relation between $\mathrm{mRNA}_{a}$ and $\mathrm{mRNA}_{b}$ is expressed as $\mathrm{mRNA}_{b}=\left(\mathrm{mRNA}_{a}\right)^{\gamma}$. Now the logarithm converts the exponential changes of the relative abundance into multiplicative factors, leading to a "multiplicative model" governing dependence between $a$ and $b: b=$ $\gamma \times a$.

It is common to assume that the multiplicative variation is imposed on top of the additive variation. This leads to the "additive-multiplicative" coherence model: $b=\lambda^{\prime}+\gamma \times a$. Fig 3 shows the effect of applying normalization preprocessing (additive coherence model) and standardization preprocessing (additive-multiplicative coherence model) on gene expression data.

A collection of microarray experiments produce a matrix with each row representing the expression profile of a gene across different timecourse or independent conditions. To maximize 
the flexibility of discovering clusters in the matrix, it is suggested that all possible combinations of coherence models on genes and/or conditions be allowed [7]. This leads to a total of 9 coherence models as shown in Table 1 .

A gene may be co-expressed via more than one coherence model. This calls for a fusion strategy that combines features corresponding to different coherence models in order to improve the prediction performance. ROCs can provide a very clear indication on which coherence models are most advantageous to fuse. For example, fig 4(a) shows that Model $(3,1)$ has a relatively higher sensitivity in the low-precision region but a relatively lower sensitivity in the high-precision region. In contrast, Model $(1,3)$ has just the opposite performance. In this case, these two coherence models are truly complementary to each other and can serve as ideal fusion candidates.

A modest fusion objective is to deliver a consistent fusion result [7], which is at least as good as any of the single model in the entire sensitivity/specificity region. As long as the sources are complementary to each other (w.r.t. the ROC), consistent fusion is always possible and it will yield improvement as long as certain statistical conditions are met. Such a goal can be achieved by a hard-switching fusion scheme as illustrated in fig 4(b).

Mathematically, denote the fusion score as $Z=$ $\alpha \phi(X)+\beta \psi(Y)$ such that the fusion output is
$g(Z)$ (c.f. Eq. 2). In the hard-switching scheme, we have either $\alpha=1, \beta=0$ or $\alpha=0, \beta=$ 1. In contrast, one may adopt a linear soft fusion scheme based on a new fusion score $Z^{\prime}=$ $\alpha^{\prime} \phi(X)+\beta^{\prime} \psi(Y)$, where $\alpha^{\prime}+\beta^{\prime}=1$. In many cases, such a soft fusion scheme can lead to betterthan-lower-bound performance. The optimal values of $\alpha^{\prime}$ and $\beta^{\prime}$ can better be derived via prominent machine learning techniques, such as Fisher classifiers and support vector machines (SVMs) with a linear kernel [10]. Unfortunately, it is known that linear classifiers often have limited discriminating power.

The most flexible decision boundaries to represent a general version of Eq. 2 can be implemented by neural networks such as SVMs or decisionbased neural networks (DBNNs) [6]. For the DBNN fusion classifier, there are two processing phases: (1) a local expert uses a Gaussian mixture model to represent the patterns of the positive (or negative) class; (2) a "gating agent" fuses the local scores to reach a Bayesian optimal decision. Fig 2(c) shows the ROCs based on such fusion scheme. Similar improvement can be found in the sensitivityspecificity ROC and the (more difficult) cluster discovery of molecular activity genes [7]. The Matlab programs that produce the results in this paper can be found in http://www.eie.polyu.edu.hk/ $\sim$ mwmak/microarray.htm. 


\section{Future Work}

It is our hope that this article will inspire more research into machine learning for genomic signal processing particularly via fusion of multiple biological or algorithmic modalities. In the near term, it should be promising to look into various machine learning techniques combining these modalities to improve prediction performance. As an example, one could first apply a parallel fusion scheme to combine coherence models $(1,3)$ and $(3,1)$, with the results being further enhanced via a cascaded fusion scheme using motifs as the feature. From a long-term perspective, the proposed fusion schemes do not have to be limited to genomic data analysis. They may be further applied to, for example, the fusion of genomic, proteomic, and transcriptomic data. Before concluding, it has to be reiterated that any computational prediction of gene clusters must be ultimately verified in the laboratory. Nevertheless, it is the authors' belief that the future advance in bioinformatics will continue to depend on a close partnership with machine learning communities.

\section{References}

[1] M. A. Beer and S. Tavazoie. Predicting gene expression from sequence. Cell, 117:185198, April 2004.

[2] Y. Cheng and G. M. Church. Biclustering of expression data. In Proc. ISMB, volume 8, pages 93-103, 2000.

[3] E. M. Conlon, X. S. Liu, J. D. Lieb, and
J. S. Liu. Integrating regulatory motif discovery and genome-wide expression analysis. PNAS, 100(6):3339-3344, March 2003.

[4] D. M. Green and J. A. Swets. Signal Detection Theory and Psychophysics. Peninsula Pub., Los Altos, Calif., 1988.

[5] J. Kasturi and R. Acharya. Clustering of diverse genomic data using information fusion. Bioinformatics, 21(4):423-429, 2005.

[6] S. Y. Kung, M. W. Mak, and S. H. Lin. Biometric Authentication: A Machine Learning Approach. Prentice Hall, Upper Saddle River, New Jersey, 2005.

[7] S. Y. Kung, M. W. Mak, and I. Tagkopoulos. Symmetric and asymmetric multimodality biclustering analysis for microarray data matrix. J. of Bioinformatics and Computational Biology, 4(3), June 2006. (In the special CSB'05 issue).

[8] P. J. Park, A. J. Butte, and I. S. Kohane. Comparing expression profiles of genes with similar promoter regions. Bioinformatics, 18(12):1576-1584, 2002.

[9] F. P. Roth, J. D. Hughes, P. W. Estep, and G. M Church. Finding DNA regulatory motifs within unaligned noncoding sequences clustered by whole-genome mRNA quantitation. Nature Biotechnology, 16:939-945, Oct. 1998.

[10] V. N. Vapnik. Statistical Learning Theory. Wiley, New York, 1998.

Acknowledgment. This work was supported in part by the Burroughs Wellcome Fund Fellowship and the Research Grant Council of Hong Kong SAR (Project No. PolyU 5230/05E). 
Table 1. Nine types of coherence models that can be obtained by applying different preprocessing methods to genes and conditions.

\begin{tabular}{|l|l||l|l|l|}
\hline \multicolumn{2}{|c||}{ Cohere Model } & \multicolumn{3}{c|}{ Condition Coherence } \\
\cline { 3 - 5 } & No adjustment & Normalization & Standardization \\
\hline \hline \multirow{3}{*}{$\begin{array}{l}\text { Gene } \\
\text { Coherence }\end{array}$} & No adjustment & Constant-value & $\begin{array}{l}\text { Mean- } \\
\text { normalization }\end{array}$ & Z-norm \\
\cline { 3 - 5 } & Normalization & $\begin{array}{l}\text { Mean- } \\
\text { normalization }\end{array}$ & $\begin{array}{l}\text { Cheng and } \\
\text { Church Type [2] }\end{array}$ & $\begin{array}{l}\text { Generalized } \\
\text { Z-norm }\end{array}$ \\
\cline { 2 - 5 } & Standardization & Z-norm & $\begin{array}{l}\text { Generalized Z- } \\
\text { norm }\end{array}$ & $\begin{array}{l}\text { Generalized } \\
\text { Z-norm }\end{array}$ \\
\hline
\end{tabular}

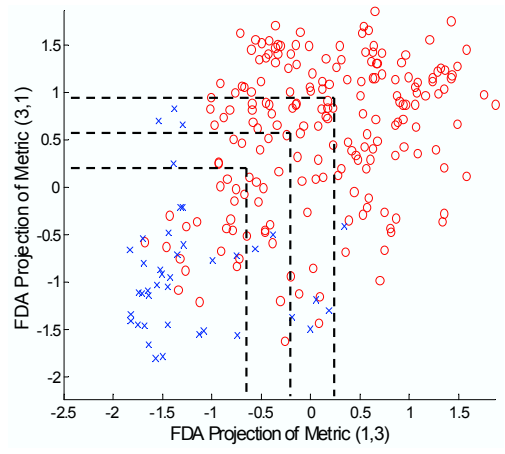

(a)

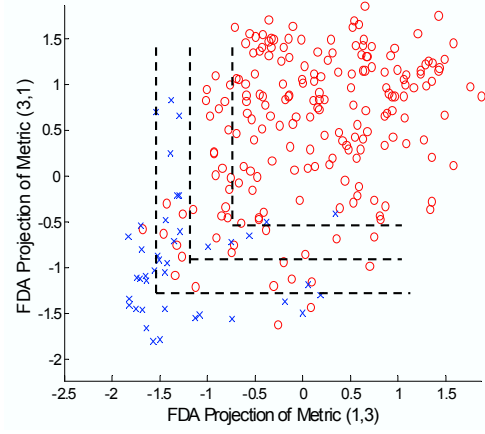

(b)

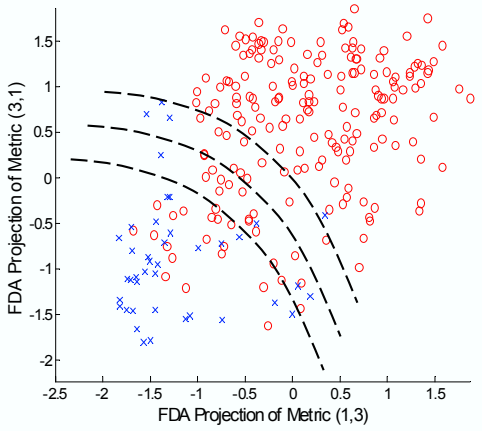

(c)

Fig. 1 Various patterns of decision boundaries created by (a) AND logic, (b) OR logic, and (c) nonlinear fusion. The patterns shown here reflect real data: blue crosses $(\times)$ represent positive (ribosomal) genes and red circle $(\circ)$ negative (non-ribosomal) genes. These examples illustrate that nonlinear fusion not only provides a unified solution but potentially improvement in prediction performance (see fig 2 ).

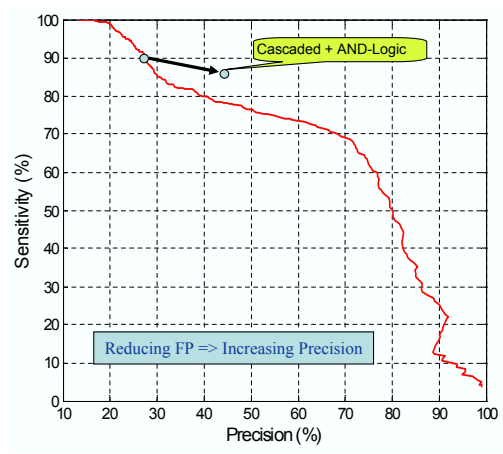

(a)

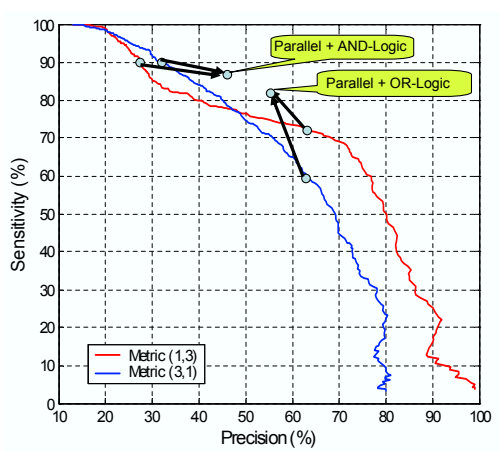

(b)

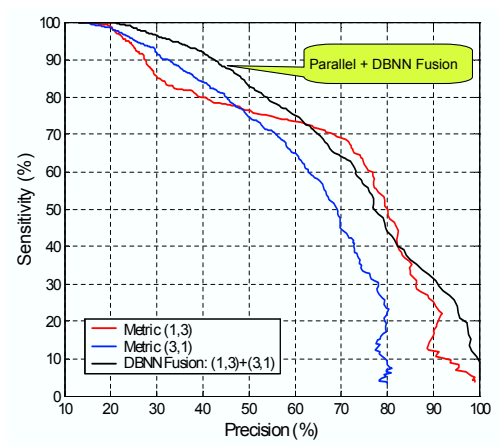

(c)

Fig. 2 Figures illustrating the benefits of cascaded and parallel fusion architectures in terms of ROCs. (a) The ROC represents the prediction performance of the first stage of cascaded fusion and the arrow indicates the possible improvement due to the AND logic in fig 1(a). For example, the AND-logic in cascaded fusion (Eq. 1) can be used to reduce the number of false positives, thus increasing the precision with a small sacrifice in sensitivity. (b) The ROCs represent the prediction performance of two modalities in parallel fusion (Eq. 2) and the arrows indicate the possible improvement due to the AND or OR logic in figures 1(a)-(b). (c) The ROC of nonlinear fusion which represents the most general scenario as shown in fig 1(c). 


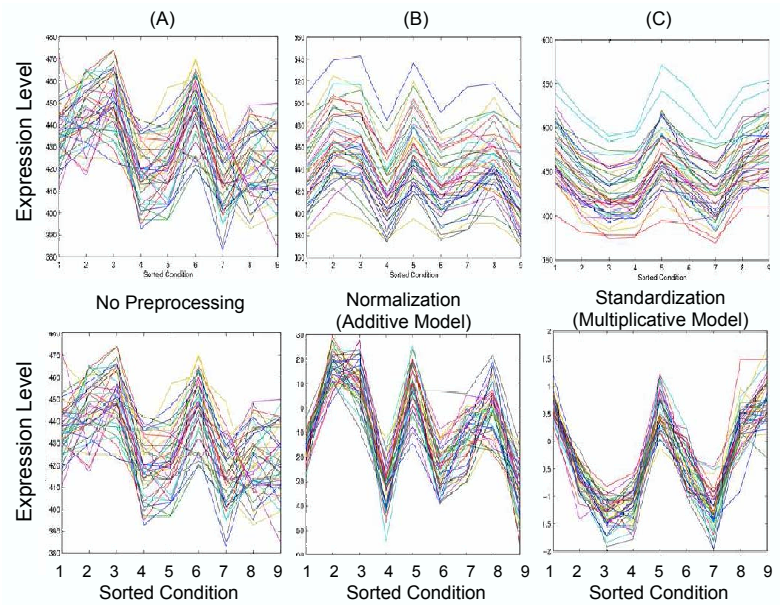

Fig. 3 Example of feature diversity. Effect of applying preprocessing on raw expression data, including normalization (additive coherence model) and standardization preprocessing (multiplicative coherence model). In the graphs, each line represents the expression level of one gene across nine conditions in the yeast data set. Shown here are three time-course gene groups: A(upper-left), B(upper-center), and C(upper-right). By the naked eye, it would not be very persuasive to claim that Group B forms a tighter family than Group A, because the former exhibits a wider spread data structure. However, if we probe into the underlying data structure, it can then be revealed that Group B has a wider spread only because there is a large variation of the additive shifts. If such an additive variation can be properly compensated by additive preprocessing, cf. lower-center-box, the remaining spread becomes relative smaller than Group A. Similarly, Group $\mathrm{C}$ will be tightened up if the underlying multiplicative variation is also compensated in addition to additive normalization, cf. lower-right-box.

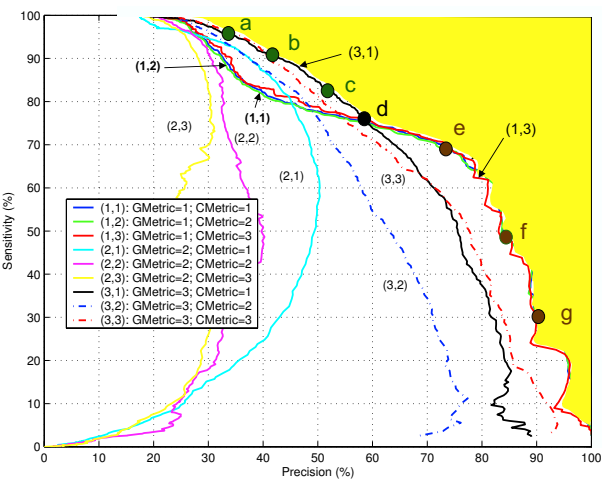

(a)

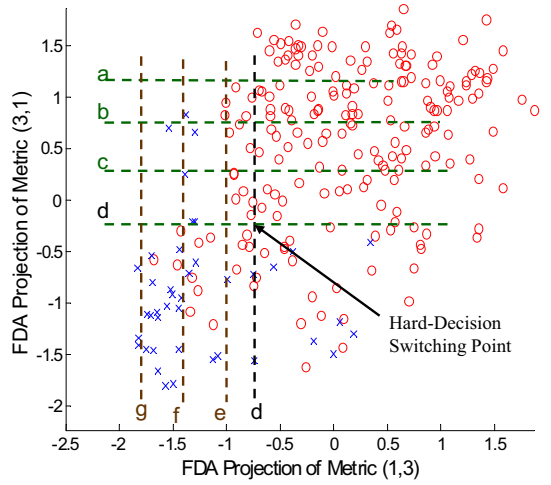

(b)

Fig. 4. (a) Performance of predicting ribosomal genes in yeast using nine different combinations of preprocessing methods for the conditions and genes. In the legend, "GMetric $=m$; CMetric $=n$ " means that coherence models $m$ and $n$ were assigned to the genes and conditions, respectively. In other words, it corresponds to Box $(m, n)$ in Table 1. The shading area represents the region of consistent fusion. (b) Decision boundaries that lead to consistent fusion. The (horizontal) decision boundaries based on Model $(3,1)$ - boundaries a, b, and c - have relatively higher sensitivity, while the (vertical) decision boundaries based on Model (3,1) - boundaries e, f, and $\mathrm{g}$ - have a relatively higher precision. Therefore, the boundaries are switched from horizontal to vertical around the crossover point. At the crossover point, boundary $\mathrm{d}$ can be either horizontal or vertical as they deliver exactly the same performance. 